\title{
Fusing Iris and Conjunctival Vasculature: Ocular Biometrics in the Visible Spectrum
}

\author{
Vikas Gottemukkula, Sashi Kanth Saripalle, \\ Sriram P. Tankasala, Reza Derakhshani \\ Dept. of Computer Science and Electrical Engineering, \\ University of Missouri - Kansas City
}

\author{
Raghunandan Pasula, Arun Ross \\ Lane Department of Computer Science and \\ Electrical Engineering, \\ West Virginia University
}

\begin{abstract}
Ocular biometrics refers to the imaging and use of characteristic features of the eyes for personal identification. Traditionally, the iris has been viewed as a powerful ocular biometric cue. However, the iris is typically imaged in the near infrared (NIR) spectrum. RGB images of the iris, acquired in the visible spectrum, offer limited biometric information for dark-colored irides. In this work, we explore the possibility of performing ocular biometric recognition in the visible spectrum by utilizing the iris in conjunction with the vasculature observed in the white of the eye. We design a weighted fusion scheme to combine the information originating from these two modalities. Experiments on a dataset of 50 subjects indicate that such a fusion scheme improves the equal error rate by a margin of 4.5\% over an iris-only approach.
\end{abstract}

Keywords: Bi-modal biometrics, RGB iris, Conjunctival vasculature.

\section{INTRODUCTION}

The science of recognizing individuals based on their unique physical or behavioral traits such as iris, fingerprints, gait and voice, etc. is termed as biometrics [1][2][3]. In this work, we consider the problem of combining iris and conjunctival vasculature paaterns (evident on the white of the eye) from ocular images acquired in the visible spectrum (i.e., RGB images). Our choice of these biometric modalities allows us to take advantage of a single acquisition device.

Though commercial iris recognition systems operate in near infrared wavelengths [4][5], given the widespread availability of color cameras, iris recognition in visible wavelengths has been of recent interest [6][7][8]. Acquisition of RGB iris images in visible spectrum also enables us to perform crossspectral matching with iris images acquired in the traditional NIR spectrum [5][9]. However, processing of RGB images acquired in the visible spectrum poses certain challenges, especially for darker colored irides resulting in poor or partially identifiable iris images. We hypothesize that this problem could be mitigated by incorporating additional information provided by the vasculature seen on the white of the eye. This vasculature comprises of episcleral and conjunctival vasculature, and is henceforth referred to as conjunctival vasculature [10][11]. The eye image acquisition system used in [10] is exclusively designed for capturing scleral pattern by asking the participants to look sideways and capturing a non-frontal ocular image. To our best knowledge this is the first attempt to fuse information from iris texture and scleral patterns from a frontal ocular image.
This work is organized as follows: Section II discusses the acquisition system and database. Section III describes the preprocessing, registration, segmentation, feature extraction and matching methods used for the analysis. The results are presented in Section IV with a detailed discussion in Section $\mathrm{V}$.

\section{DATA ACQUISITION}

With the aim of using a single acquisition unit for acquiring both the iris and the conjunctival vasculature simultaneously in the visible spectrum, we designed a hyperfocal image acquisition system for this study.

Under UMKC IRB protocol 11-57e, fifty volunteers (22 male, 28 female, ages 19 to 59 years) were invited for two data capture sessions 4-30 days apart. During each session, for each subject, two series of captures were acquired with a time lapse of 30-45 minutes between captures. 47 volunteers participated in both sessions yielding 194 series of captures. The volunteers were asked to open their eyes and look directly into the camera lens during data acquisition, where the entire face is captured at a resolution of $2832 \times 4256$. A Nikon D3S FX format camera with 12.1 effective megapixels and a micro Nikkor $105 \mathrm{~mm}$ lens were used for data capture. The camera was operated in the "program" mode where aperture and shutter parameters are automatically determined. However, for all the captures, the f-number was less than 5 . Based on the pilot studies, we used an ISO of 1000 for best simultaneous capture of conjunctival and iridial patterns. For lighting, we used a front ring light (Digi-Slave flex ring 6400) and blocked ambient light by asking the participants to place their face inside a box lined with velvet cloth. Additional pre-flash red LEDs were used to reduce the dilation of pupil. The acquisition system along with the lighting setup is shown in Figure 1. While the acquisition system may be viewed as being highly controlled, our goal is to first establish the possibility of combining the iris and the conjunctival vasculature.

\section{FEATURE EXTRACTION AND MATCHING METHODS}

\section{A. Iris Recognition}

Ocular regions corresponding to the left and right eyes were manually cropped from every face image. Figure 2 shows the manually cropped ocular regions for a subject in the dataset. 


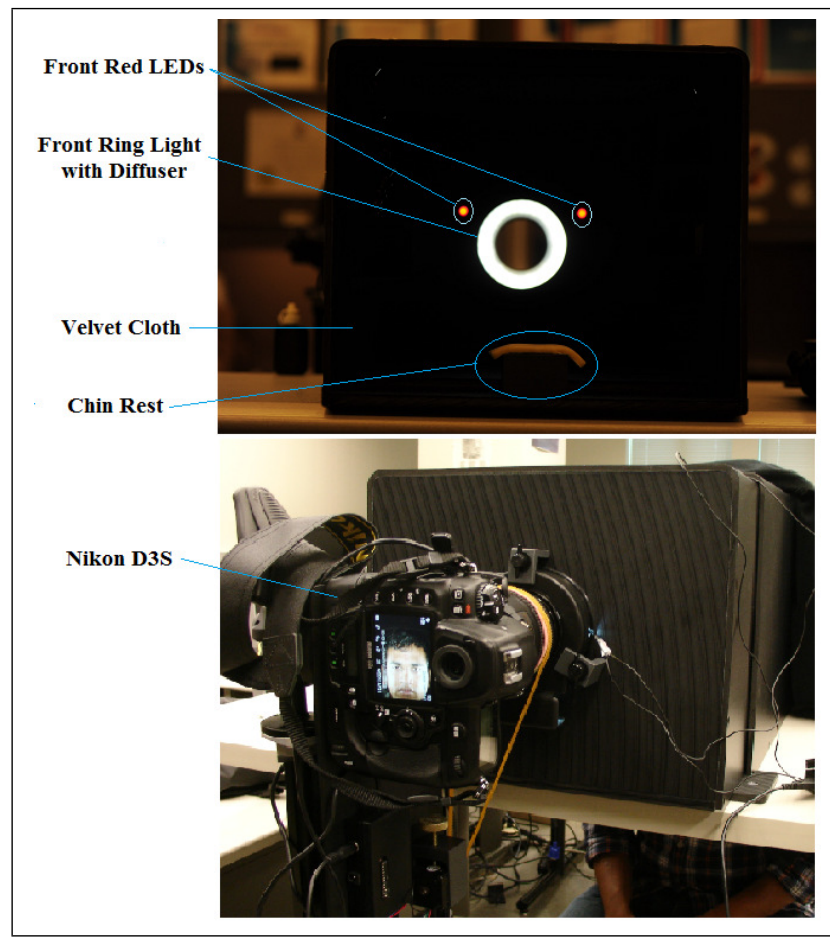

Fig. 1. Components of the data acquisition system.

The irides in the entire dataset had an average diameter of 208 pixels.

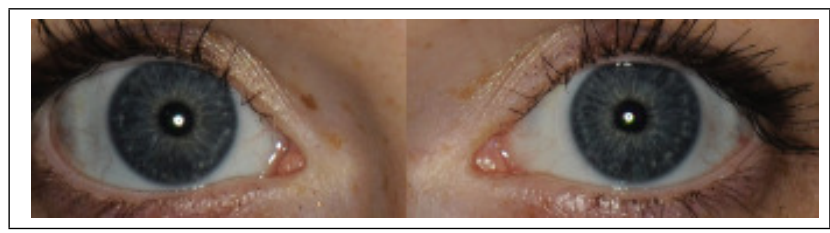

Fig. 2. Right and left ocular regions.

Most of the irides in the dataset are dark brown to black. There is minimal textural clarity in the dark colored iris images, since they are captured in the visible spectrum. It is also observed that there is no clear contrast between the pupillary region and the iris region in such images. In this work, the red channel was used for the iris modality since it offers discernible information due to its proximity to the near infrared channel. Figure 3 shows the RGB and red channel image for a dark colored iris and a light colored iris. Integro-differential operator [3] is used to detect the pupillary and limbic boundaries. The upper and lower eyelids are approximated using horizontal lines. Incorrectly segmented iris images are manually detected and segmented. Since the goal of this work is to assess the benefits of combining the iris with the conjunctival vasculature, the effects of incorrect segmentation are mitigated by manual intervention. Once the iris region is segmented, it is normalized into a fixed size rectangular grid using Daugman's rubber sheet model [3]. The masked regions in the iris region corresponding to eye lids and specular reflections are summarized in a binary image referred to as a mask image (Figure 4 (d)). Zeros (black pixels) correspond to the true iris texture and ones (white pixels) correspond to occluded pixels in Figure 4. 1-D log Gabor filters are applied to rows of the normalized image in the Fourier domain and inverse Fourier transform is applied to the filtered response to result in a complex valued output. The frequency response of a Log-Gabor filter is given as

$$
G(f)=\exp \left[\frac{-\left(\log \left(f / f_{o}\right)\right)^{2}}{2\left(\log \left(\sigma / f_{o}\right)^{2}\right.}\right]
$$

where, $f_{o}$ is the center frequency and $\sigma$ is the bandwidth of the filter.

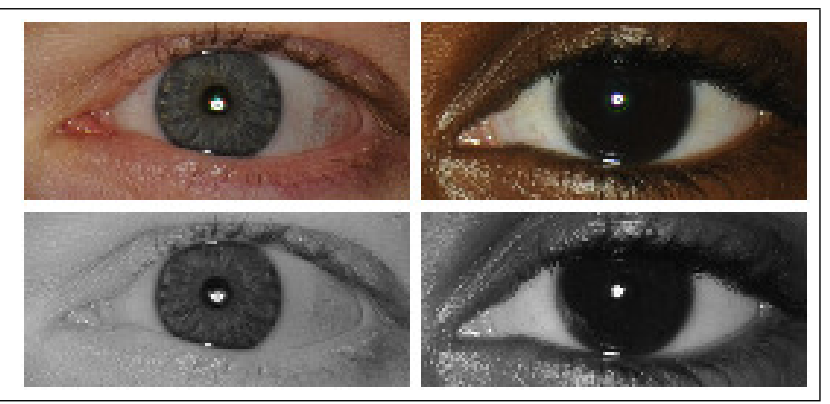

Fig. 3. The images on the top correspond to a light iris (left) and a dark iris (right). The images on the bottom are the corresponding red channel images.

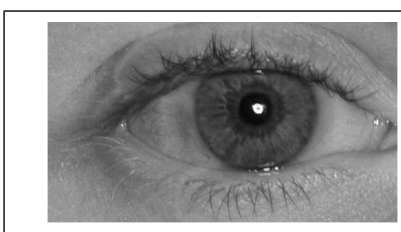

(a)

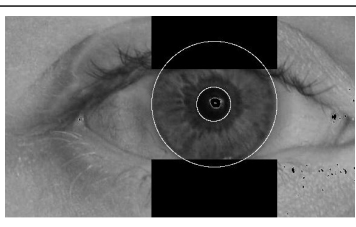

(b)

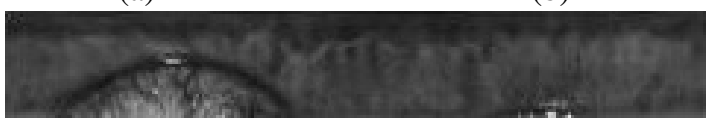

(c)

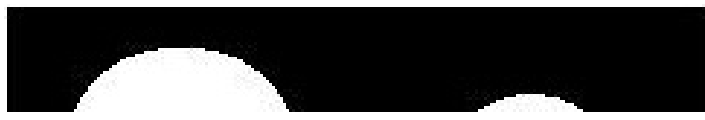

(d)

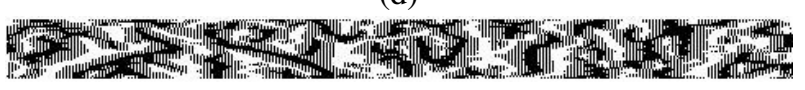

(e)

Fig. 4. (a) Original image (b) Segmentation output: detection of pupillary (inner) and limbic (outer) boundaries of the iris (c) Normalized iris (d) Mask image and (e) Corresponding IrisCode.

The output is then quantized into 0's and 1's using Daugman's method [3]. This process converts a normalized image into a binary sequence of 0 's and 1's, referred to as 'IrisCode'. The outputs of the steps involved in generating an IrisCode is shown in Figure 4. The reader is referred to [3] for a detailed description of IrisCodes. 
Hamming distance is used to measure the dissimilarity between two IrisCodes. Hamming distance between two irides $\mathrm{A}$ and $\mathrm{B}$, whose IrisCodes are codeA and $\operatorname{codeB}$, respectively, and whose masks are maskA and maskB, respectively, is given by:

$$
d=H D=\frac{\|(\operatorname{code} A \otimes \operatorname{code} B) \bigcap \text { mask } A \bigcap \text { mask } B \|}{\| \text { mask } A \bigcap \text { mask } B \| .}
$$

The above formula computes the average dissimilarity between two binary IrisCodes in the unmasked regions. After generating the match score for left and right iris separately we used the simple sum rule to fuse the scores.

\section{B. Conjunctival Vasculature Recognition}

1) Image Registration: The Integro-differential circle finder method, used in iris recognition, is used to find the radius and center of the iris as a reference landmark for the sclera of the eye. The green layer of the RGB image is used to process the sclera and the accompanying vasculature. After calculating the centers of both irides, the angle artifact in the coronal plane, due to tilted faces, is removed (Figure 5). This improves the registration of the square tiles (described in the next section). To do so, the angle of the line passing through the two iridial centers is measured with respect to the x-axis, and the image is rotated to reduce this angle to zero. Then, the new iris center coordinates $(\mathrm{X}, \mathrm{Y})$ are determined using the rotation matrix $(\mathrm{RM})$ and the center of the image coordinates $\left(X_{C}, Y_{C}\right)$ as:

$$
\begin{gathered}
R M=\left[\begin{array}{cc}
\cos \theta & -\sin \theta \\
\sin \theta & \cos \theta
\end{array}\right] \\
{[X Y]=\left(R M \times\left(\left[\begin{array}{l}
x \\
y
\end{array}\right]-\left[\begin{array}{l}
X_{C} \\
Y_{C}
\end{array}\right]\right)\right)^{\prime}+\left[\begin{array}{ll}
X_{C} & Y_{C}
\end{array}\right]}
\end{gathered}
$$

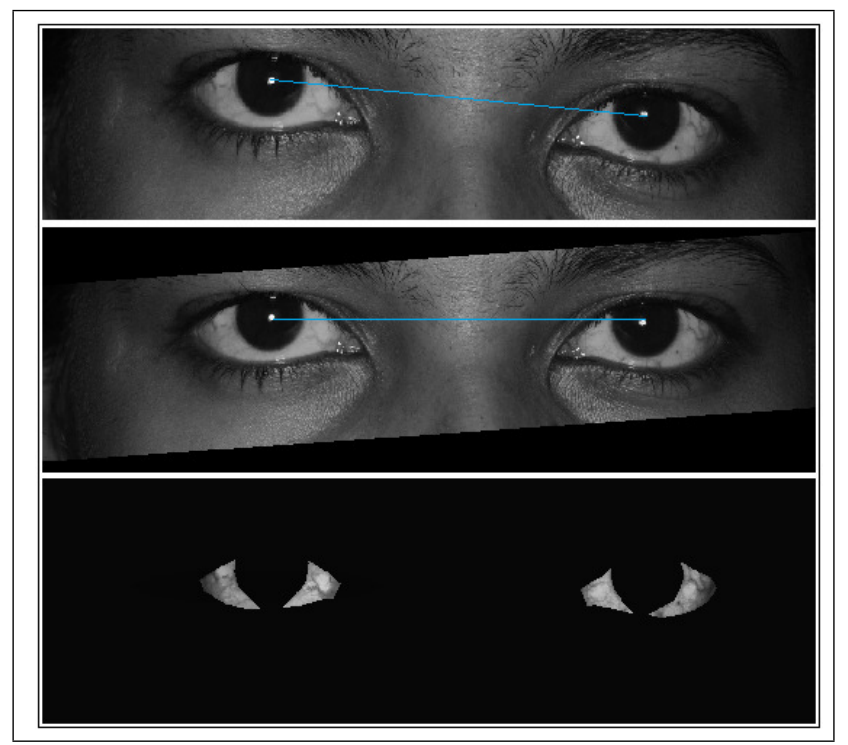

Fig. 5. Top: The green channel of an image showing the face angle artifact with respect to coronal plane. Middle: Angle-compensated image. Bottom: CLAHE applied to conjunctival region.
2) Tiling and Feature Extraction: The amount of vasculature available is critical to the matching performance due to the conjunctival vasculature. Furthermore, due to variation in eye openings and eyelid contours across images, as well as the presence of affine artifacts, it is challenging to register the vascular regions of interests extracted from segmented scleral areas. Hence, we designed a scleral image tiling technique for frontal (straight) gaze images. The new tiling technique considers the iris center and boundary as a spatial reference, and places square tiles around the iris, with sides proportional to the respective iridial radius. In order to account for interand intra-subject iris radius variations caused due to, for instance, variations in positioning of the face with respect to the camera, the side of the tiling element was set proportional to the iris radius. In a single image, we used the average radii from both irides to better determine the aforesaid tile size. By a process of trial and error, we observed two tile sizes to provide best overall results: one third and one fourth of the iridial radius. Similarly, we established two different tile placement patterns for both tile sizes (Figure 6). The aforesaid placements use 160 tiles when the tile side is one third of iridial radius, and 260 tiles when the tile side is one fourth of iridial radius.

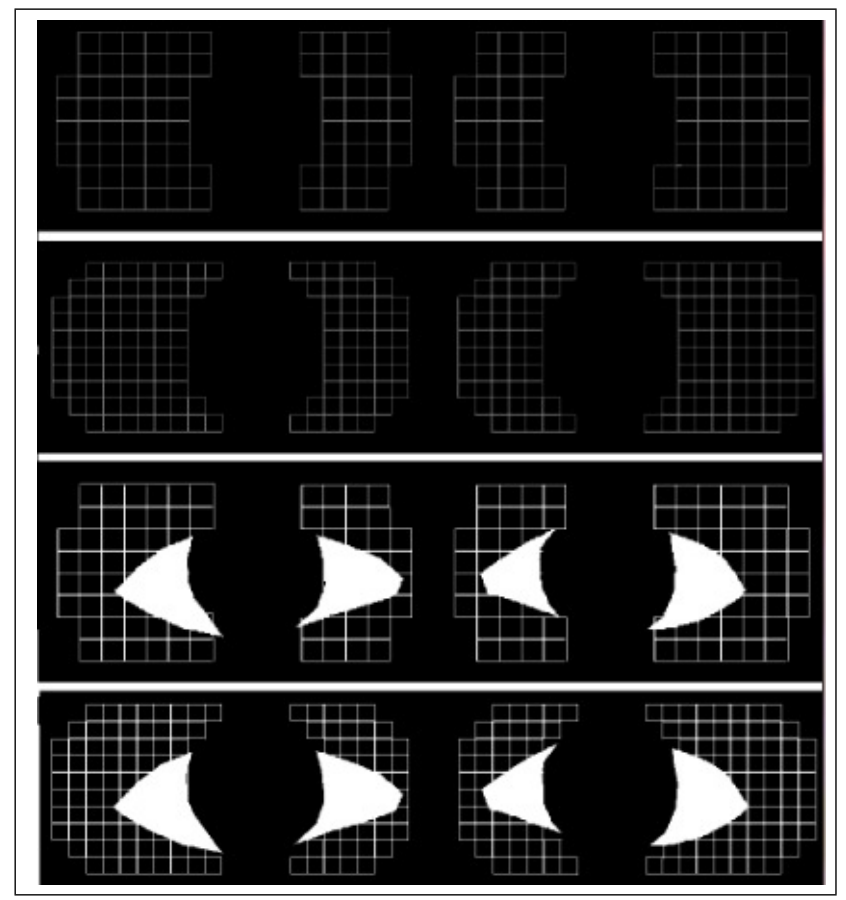

Fig. 6. From top: 1) Tile placement when tile side is one third the iridial radius. 2) Tile placement when each side is one fourth the iridial radius. 3) and 4) The tile-grid overlaid on the scleral mask.

For our analysis, we used masks obtained by manual tracing of the scleral contours. For image preprocessing, we applied contrast limited adaptive histogram equalization (CLAHE) on the green (red-free) layer of each conjunctival image (Figure $5)$. The mean and variance of each of the enhanced valid tile pixels are then taken as simple experimental texture features. 
Two feature vectors, one obtained by concatenating the mean values and the other obtained by concatenating the variance values, are generated. A tile is deemed valid if at least $80 \%$ of its area is within the scleral mask. A match score is generated by adding the correlation coefficient between the two feature vectors.

3) Fusion: Each RGB eye image contains both iris and conjunctival vascular information. Iris matching results in a set of dissimilarity scores (i.e. Hamming distance, d; lower score is a better match) and conjunctival matching results in a set of similarity scores (correlation coefficient $r$; higher score is a better match). A fusion scheme is implemented to combine these scores and analyze the possibility of increased performance.

First, iris dissimilarity score, d, is converted to a similarity score, s, such that $s=1-d$. Next, conjunctival and the iris similarity scores are normalized using the min-max rule to ensure that they are in the $[0,1]$ interval. Normalized similarity scores corresponding to iris and conjunctival matching are then fused using a simple sum rule.

We also implemented a weighted fusion method where scores from iris and conjunctival vasculature modalities are multiplied by a weighting factor before they are added.

$S_{\text {Fused }}=w_{1} * S_{\text {Leftiris }}+w_{2} * S_{\text {Rightiris }}+w_{3} * S_{\text {Vasculature }}$

Here $w_{1}+w_{2}+w_{3}=1$

\section{RESULTS}

For all the subjects, two samples from Session 1 and two samples from Session 2 are used to generate the final set of matching scores. The two samples from each session have a time lapse of 30-45 minutes. Thus, a total of 4 samples for each of the 47 subjects who finished both sessions and 2 samples for each of the 3 subjects who finished only the first session are used to generate the match scores. Receiver Operating Characteristic (ROC) curves were generated to observe the performance of the system. The following four ROC curves are generated: 1) training with Session 1 samples and testing with Session 1 samples (short term analysis 1: 50 subjects with 100 genuine and 9800 imposter scores), 2) training with Session 2 sample and testing with Session 2 samples (short term analysis 2: 47 subjects with 94 genuine and 8648 imposter scores), 3) training with Session 1 sample and testing with Session 2 samples and vice versa (long-term term analysis: 376 genuine and 18424 imposter scores), and 4) all-pairs matching (570 genuine and 36872 imposter scores).

\section{A. Iris Recognition}

The results of iris recognition in the above four cases for right and left irides are presented in Table I and the corresponding ROCs are plotted in Figures 7 and 8. IrisCode is partially invariant to changes in illumination and angle of acquisition. However, as the images are acquired in the visible spectrum, the darker irides exhibit low textural clarity. Combined with the fact that pupillary boundary for few iris images could not be manually determined, the EERs for iris recognition are higher compared to the traditional performance of iris images acquired in NIR spectrum. An overall EER of $6.87 \%$ was achieved for the right iris and $9.5 \%$ for the left iris in this dataset.

TABLE I

PERFormance EVALUATION OF LEFT AND RIGHT IRIS

\begin{tabular}{|c|c|c|}
\hline \multirow{2}{*}{ Analysis } & \multicolumn{2}{|c|}{ EER } \\
\cline { 2 - 3 } & Left iris & Right iris \\
\hline Session1 & $7.6 \%$ & $9.37 \%$ \\
\hline Session2 & $4.6 \%$ & $4.29 \%$ \\
\hline $\begin{array}{l}\text { Session1 vs Session2 } \\
\text { Session2 vs Session1 }\end{array}$ & $11.4 \%$ & $6.82 \%$ \\
\hline All-pairs matching & $9.5 \%$ & $6.87 \%$ \\
\hline
\end{tabular}

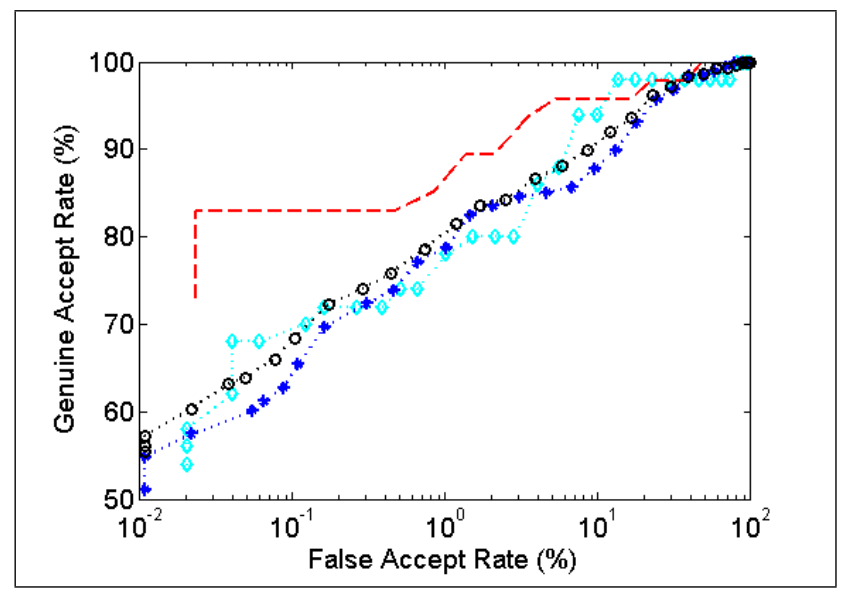

Fig. 7. ROC plots of left iris recognition. Cyan - Session 1 vs. Session 1 samples; Red - Session 2 vs. Session 2 samples; Blue - Session 1 vs. Session 2 samples and Session 2 vs. Session 1 samples; Black - All-pairs matching

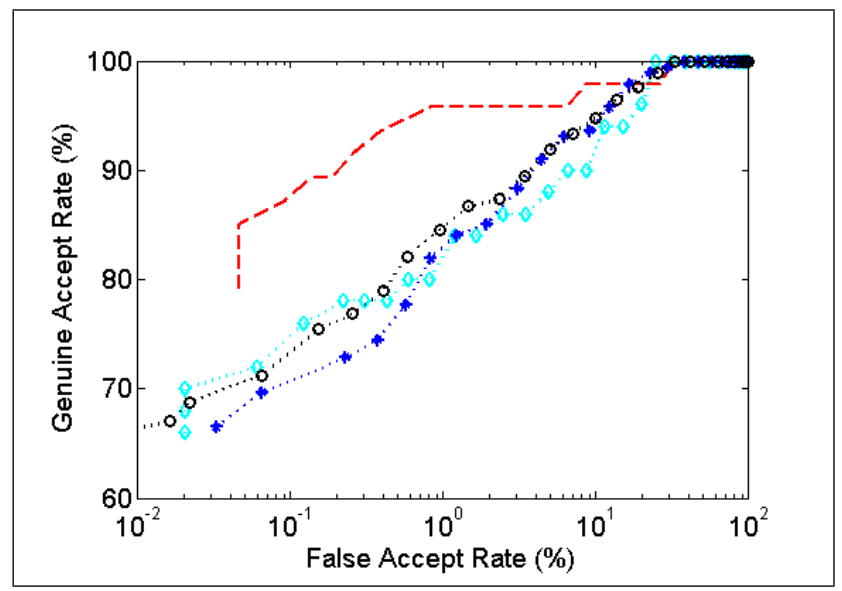

Fig. 8. ROC plots of right iris recognition. Cyan - Session 1 vs. Session 1 samples; Red - Session 2 vs. Session 2 samples; Blue - Session 1 vs. Session 2 samples and Session 2 vs. Session 1 samples; Black - All-pairs matching

\section{B. Conjunctival Vasculature Recognition}

Based on empirical evaluation, we eventually used a tile size of one-fourth iridial radius, and extracted mean and variance of 
pixel values within each tile. The mean values of all the tiles were used to create a feature vector; similarly the variance values of all the tiles were used to create another feature vector. The corresponding feature vectors of the left and right eyes were concatenated resulting in two super vectors- one corresponding to the mean values and the other corresponding to the variance values. Correlation coefficient was used to assess the similarity between two super vectors corresponding to two different images. The sum of the two correlation coefficients was then used to generate the match score. When system is trained and tested with the same day samples (short term analysis), EERs ranged between 4-6\%. As expected, these figures deteriorated for the long term study (Figure 9). Nonetheless, the benifit of the proposed fusion becomes more apparent when the scores originating from the other ocular biometric modality extracted from the same set of images, i.e. the RGB iris image, are fused with conjunctival match scores.

TABLE II

Performance EVAluation OF CONJUNCTIVAl VASCUlature

\begin{tabular}{|c|c|}
\hline Analysis & EER \\
\hline Session1 & $6.01 \%$ \\
\hline Session2 & $4.01 \%$ \\
\hline $\begin{array}{l}\text { Session1 vs Session2 } \\
\text { Session2 vs Session1 }\end{array}$ & $13.91 \%$ \\
\hline All-pairs matching & $11.28 \%$ \\
\hline
\end{tabular}

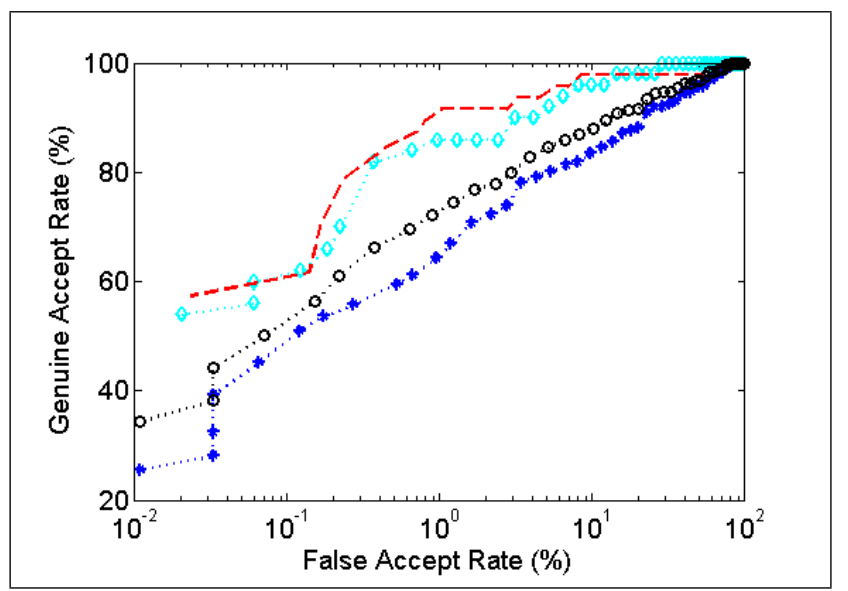

Fig. 9. ROC plots of conjunctival vasculature recognition. Cyan - Session 1 vs. Session 1 samples; Red - Session 2 vs. Session 2 samples; Blue - Session 1 vs. Session 2 samples and Session 2 vs. Session 1 samples; Black - All-pairs matching

\section{Fusion}

The EERs for the simple sum rule fusion scheme are presented in Table III and the corresponding ROCs are plotted in Figure 10. It is observed that there is a considerable increase in performance after fusion using the simple sum rule. The fusion resulted in a much improved EER of $2.83 \%$ compared to $6.87 \%$ for right iris, $9.5 \%$ for left iris and $11.28 \%$ for conjunctival matching.
TABLE III

PERFORMANCE EVALUATION OF IRIS AND CONJUNCTIVAL VASCULATURE USING SIMPLE SUM RULE

\begin{tabular}{|c|c|}
\hline Analysis & EER \\
\hline Session1 & $1.5 \%$ \\
\hline Session2 & $0.55 \%$ \\
\hline $\begin{array}{c}\text { Session1 vs Session2 } \\
\text { Session2 vs Session1 }\end{array}$ & $4.05 \%$ \\
\hline All-pairs matching & $2.83 \%$ \\
\hline
\end{tabular}

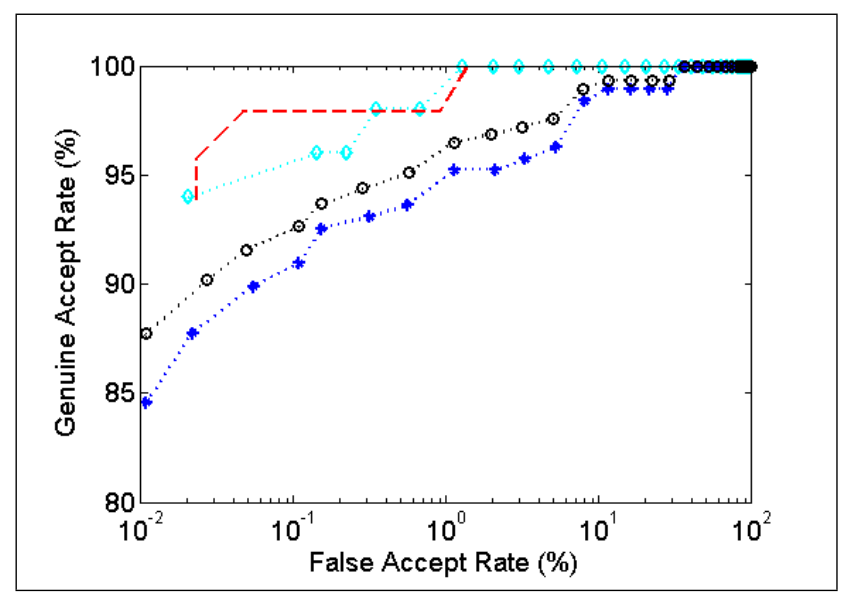

Fig. 10. ROC plots after fusing conjunctival match scores with left and right iris match scores using simple sum rule. Cyan - Session 1 vs. Session 1 samples; Red - Session 2 vs. Session 2 samples; Blue - Session 1 vs. Session 2 samples and Session 2 vs. Session 1 samples; Black - All-pairs matching

Various weights were assigned to the individual modalities, and the best, based on the lowest EER on the entire dataset, is tabulated in Table IV and the corresponding ROC plots are in Figure 11. Weighted fusion improves the overall results compared to the simple sum rule fusion. Here, conjunctival vasculature, left iris and right iris scores are added with weights of $0.25,0.39$ and 0.36 , respectively. Table $\mathrm{V}$ shows EERs obtained using different combinations of weights for different mobilities.

TABLE IV

Performance EVAluation When USING Weighted Linear Fusion of IRIS AND CONJUNCTIVAL MATCH SCORES

\begin{tabular}{|c|c|}
\hline Analysis & EER \\
\hline Session1 & $1.92 \%$ \\
\hline Session2 & $0.53 \%$ \\
\hline $\begin{array}{l}\text { Session1 vs Session2 } \\
\text { Session2 vs Session1 }\end{array}$ & $3.71 \%$ \\
\hline All-pairs matching & $2.39 \%$ \\
\hline
\end{tabular}

\section{Discussion}

We noticed that the selection of tile size plays an important role in determining matching performance due to the conjunctival modality. When using bigger tiles we have less features, but translational variations between images due to imaging artifacts are reduced. On the other hand, with smaller tiles, precise registration is essential, and there is a possibility of incorrectly including more vascular tiles; however the 


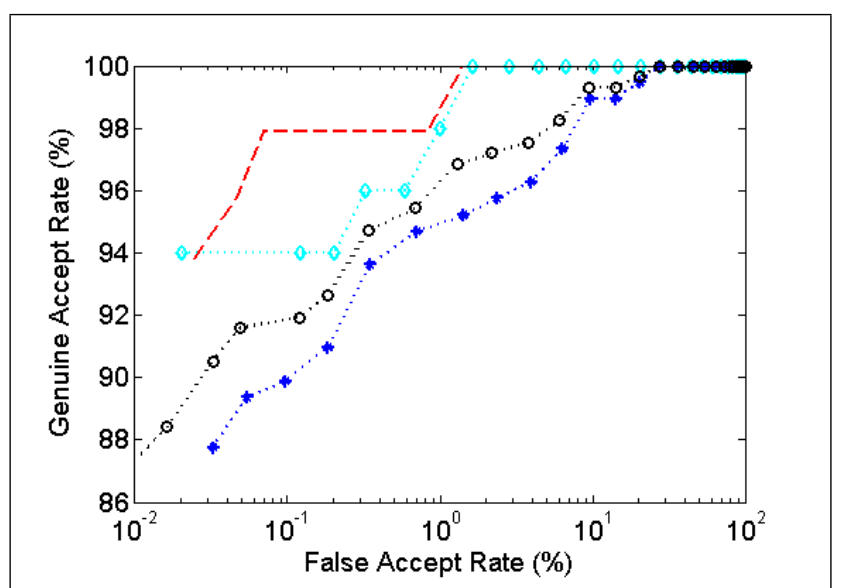

Fig. 11. ROC plots of weight based fusion. Cyan - Session 1 vs. Session 1 samples; Red - Session 2 vs. Session 2 samples; Blue - Session 1 vs. Session 2 samples and Session 2 vs. Session 1 samples; Black - All-pairs matching

TABLE V

PERFormanCE EVALUATION OF WEIGHTED FUSION ON THE ENTIRE DATASET

\begin{tabular}{|c|c|c|c|}
\hline Left Iris & Right Iris & Conjunctival Vasculature & EER \\
\hline 1 & 0 & 0 & $9.5 \%$ \\
\hline 0 & 1 & 0 & $6.87 \%$ \\
\hline 0 & 0 & 1 & $11.28 \%$ \\
\hline 0.5 & 0.5 & 0 & $4.7 \%$ \\
\hline 0.33 & 0.33 & 0.33 & $2.83 \%$ \\
\hline $\mathbf{0 . 3 9}$ & $\mathbf{0 . 3 6}$ & $\mathbf{0 . 2 5}$ & $\mathbf{2 . 3 9 \%}$ \\
\hline
\end{tabular}

resolution of feature vectors, in this case, is increased. The current set of feature vectors used a tile size corresponding to $1 / 4$ of iris radius.

Fusion of both modalities based on the sum rule and the weighted sum rule significantly improves the results. This research establishes the benefits of fusing scleral information with the iris in RGB images of the ocular region. Unlike periocular biometrics [12], this bimodal configuration explicitly uses the iris and requires only a small portion of the ocular region.

\section{AcKnowledgment}

Authors are grateful for the mechanical design done by Bret Lesan, CMfgE, Senior Research and Design Engineer, University of Missouri Kansas City, USA. Authors are thankful to Plamen Doynov for his suggestions on the abstract. Research was sponsored by the Leonard Wood Institute in cooperation with the U.S. Army Research laboratory and was accomplished under Cooperative Agreement Number W911NF-072-0062. The views and conclusions contained in this document are those of the authors and should not be interpreted as representing the official policies, either expressed or implied, of the Leonard Wood Institute, the Army Research Laboratory or the U.S. Government. The U.S. Government is authorized to reproduce and distribute reprints for Government Purposes not withstanding any copyright notation hereon.

\section{REFERENCES}

[1] A. K. Jain, A. Ross, and S. Prabhakar, "An introduction to biometric recognition," IEEE Transactions on Circuits and Sytems for Video Technology, vol. 14, no. 1, pp. 4-20, 2004.

[2] A. K. Jain, P. J. Flynn, and A. A. Ross, "Handbook of biometrics," Springer, 2007.

[3] J. Daugman, "How iris recognition works," IEEE Transactions on Circuits and Systems for Video Technology, vol. 14, no. 1, pp. 21-30, 2004.

[4] K. Bowyer, K. Hollingsworth, and P. Flynn, "Image understanding for iris biometrics: A survey," Computer Vision and Image Understanding, vol. 110, no. 2, pp. 281-307, 2008.

[5] C. Boyce, A. Ross, M. Monaco, L. Hornak, and X. Li, "Multispectral iris analysis: A preliminary study," in IEEE Computer Vision and Pattern Recognition Workshop, p. 51, 2006.

[6] Proença, "Iris recognition: On the segmentation of degraded images acquired in the visible wavelength," IEEE Transactions on Pattern Anlysis and Machine Intelligence, vol. 32, no. 8, pp. 1502-1516, 2010.

[7] H. Proença and L. Alexandre, "Ubiris: A noisy iris image database," ICIAP, pp. 970-977, 2005.

[8] A. Ross, "Iris recognition: The path forward," Computer, vol. 43, no. 2, pp. $30-35,2010$.

[9] A. Ross, R. Pasula, and L. Hornak, "Exploring multispectral iris recognition beyond 900nm," in IEEE 3rd International Conference on Biometrics: Theory, Applications, and Systems, pp. 1-8, 2009.

[10] S. Crihalmeanu and A. Ross, "Multispectral scleral patterns for ocular biometric recognition," Pattern Recognition Letters, 2012.

[11] R. Derakhshani and A. Ross, "A texture-based neural networks classifier for biometric identification using ocular surface vasculature," IEEE International Conference on Neural Networks, pp. 2982-2987, 2007.

[12] U. Park, R. R. Jillela, A. Ross, and A. K. Jain, "Periocular biometrics in the visible spectrum," IEEE Transactions on Information Forensics and Security, vol. 6, no. 1, pp. 96-106, 2011. 